\title{
Complete Band Gaps in One-Dimensional Left-Handed Periodic Structures
}

\author{
Ilya V. Shadrivov, Andrey A. Sukhorukov, and Yuri S. Kivshar \\ Nonlinear Physics Centre and Centre for Ultrahigh Bandwidth Devices for Optical Systems, \\ Research School of Physical Sciences and Engineering, Australian National University, \\ Canberra ACT 0200, Australia
}

(Received 17 March 2005; published 2 November 2005)

\begin{abstract}
Artificially fabricated structures with periodically modulated parameters such as photonic crystals offer novel ways of controlling the flow of light due to the existence of a range of forbidden frequencies associated with a photonic band gap. It is believed that modulation of the refractive index in all three spatial dimensions is required to open a complete band gap and prevent the propagation of electromagnetic waves in all directions. Here we reveal that, in sharp contrast to what was known before and contrary to the accepted physical intuition, a one-dimensional periodic structure containing the layers of transparent left-handed (or negative-index) metamaterial can trap light in three-dimensional space due to the existence of a complete band gap.
\end{abstract}

PACS numbers: 42.70.Qs, 41.20.Jb, 78.67.Pt

Photonic crystals are artificial materials with a periodic modulation in the dielectric constant which can create a range of forbidden frequencies called a photonic band gap [1]. Photons with frequencies within the band gap cannot propagate through the medium. This unique feature can alter dramatically the properties of light, enabling control of spontaneous emission in quantum devices and light manipulation for photonic information technology [2]. Photonic band-gap structures can also be found in nature, and they explain the color diversity of some of the living creatures [3].

Complete two-dimensional (2D) and three-dimensional (3D) band gaps can be realized in photonic crystals, where the refractive index is periodically modulated in two or three dimensions, respectively [1]. Such modulation is necessary to satisfy the Bragg condition simultaneously for all propagation directions, requiring that phase accumulation per period is close to a multiple of $\pi$, so that the waves reflected at different interfaces between the regions with low and high refractive indexes interfere constructively and wave propagation is prohibited for any incidence angle. Manufacturing of 3D photonic crystals still remains a technological challenge due to the requirements of large index contrast and high fabrication precision.

The simplest periodic structure, both in geometry and manufacturing, is a one-dimensional stack of two types of layers which differ in the dielectric constant [4]. However, such structures may only possess partial band gaps for certain ranges of propagation directions. Here we study the scattering properties of one-dimensional periodic structures containing layers made of the so-called left-handed metamaterials (LHMs) - artificially created composites which are characterized by simultaneously negative dielectric permittivity and magnetic permeability. Such materials are transparent and can bend light in the opposite direction to normal reversing the way in which refraction usually works [5]. We demonstrate that specially designed onedimensional structures with negative refraction may ex- hibit a complete three-dimensional band gap. First, we show that a layered structure made of two alternating layers of left-handed materials and conventional dielectrics can exhibit a complete two-dimensional band gap; i.e., it does not support any propagating TE or TM waves and is therefore opaque for any angle of propagation in the plane. As a direct consequence of such band gap, the radiation of waves with given polarization by any source placed anywhere inside this structure is prohibited, and the twodimensional density of states (DOS) [6] is zero. This result is in sharp contrast to directional reflection [7-9] in conventional layered dielectric structures, which reflect only electromagnetic waves launched from air or a low-index medium. In the periodic structures with usual dielectrics a source dipole can emit radiation of both polarizations, indicating that the complete gap is absent and propagating TE and TM modes are always present. The outcoupling of radiation from a periodic structure can vanish only at certain interfaces [see Ref. [10] and references therein], whereas the radiation along the layers, as well as DOS, is never zero. We reveal the physical effects which lead to such fundamental differences between periodic structures with conventional and left-handed layers. In the final part of the Letter, we suggest a design of a one-dimensional periodic structure consisting of three alternating layers made of conventional dielectric and left-handed materials which possesses a complete three-dimensional band gap.

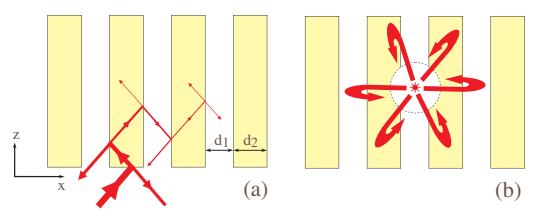

FIG. 1 (color online). Schematic of the left-handed periodic structure with (a) the ray diagram for the end-fire wave propagation and (b) suppression of radiation of a local source placed inside. 
We consider a one-dimensional periodic structure created by layers (with the thicknesses $d_{1}$ and $d_{2}$ ) of two different materials with dielectric permittivities $\epsilon_{1,2}$ and magnetic permeabilities $\mu_{1,2}$, respectively, as shown in Fig. 1. First, we study the propagation of the TE-polarized electromagnetic waves which have the component of the electric field parallel to the layers $\left(E=E_{y}\right)$; all results can be easily generalized for the case of the TM-polarized waves. We consider the wave propagation in the $(x, z)$ plane characterized by the wave vector $\mathbf{k}=\left(k_{x}, 0, k_{z}\right)$. The TE-polarized waves are described by the linear Helmholtz-type equation for the electric field component,

$$
\Delta E+\frac{\omega^{2}}{c^{2}} n^{2}(x) E-\frac{1}{\mu(x)} \frac{\partial \mu}{\partial x} \frac{\partial E}{\partial x}=0,
$$

where $\Delta=\partial^{2} / \partial x^{2}+\partial^{2} / \partial z^{2}$ is the two-dimensional Laplacian and $n^{2}(x)=\epsilon(x) \mu(x)$ is the square of refractive index. In a one-dimensional periodic structure the propagating waves have the form of Bloch modes, for which the electric field amplitudes satisfy the periodicity condition, $E(x+\Lambda, z)=E(x, 0) \exp \left(i K_{b}+i k_{z} z\right)$, where $\Lambda=d_{1}+d_{2}$ is the period of the structure. Here $K_{b}$ is the dimensionless Bloch wave number which defines the wave transmission across the layers, and its dependence on the wave vector component along the layers $\left(k_{z}\right)$ can be found explicitly for two-layered periodic structures [see, e.g., Refs. [4,11]],

$$
\begin{aligned}
2 \cos \left(K_{b}\right)= & \operatorname{Tr}(M) \\
= & 2 \cos \left(k_{1 x} d_{1}\right) \cos \left(k_{2 x} d_{2}\right) \\
& -\left(\frac{k_{2 x} \mu_{1}}{k_{1 x} \mu_{2}}+\frac{k_{1 x} \mu_{2}}{k_{2 x} \mu_{1}}\right) \sin \left(k_{1 x} d_{1}\right) \sin \left(k_{2 x} d_{2}\right) .
\end{aligned}
$$

Here $\operatorname{Tr}(M)$ is the trace of the transfer matrix $M$ characterizing the wave scattering in a periodic structure [4], $k_{j x}=$ $k_{j}\left(1-k_{z}^{2} / k_{j}^{2}\right)^{1 / 2}$ are the $x$ components of the wave vector in the first $(j=1)$ and second $(j=2)$ media, and $k_{j}=$ $\omega n_{j} / c$ are wave numbers in each media with refractive indexes $n_{j}$. For completeness, we mention that the dispersion relation of the TM-polarized waves is obtained by replacing $\epsilon \Leftrightarrow \mu$ in Eq. (2).

Solutions of the dispersion relation (2) with both real $k_{z}$ and $K_{b}$ correspond to Bloch waves which can propagate through the periodic structure, whereas complex $k_{z}$ or $K_{b}$ indicate the presence of band gaps in the spectrum where the wave propagation is prohibited. A complete band gap occurs if for all real $k_{z}$, the $K_{b}$ remains complex. It was recently shown $[11,12]$ that novel partial band gaps can appear in structures made of alternating layers of LHM and normal dielectrics when the condition of zero average refractive index is satisfied for particular propagation angles $\left(k_{z}\right), k_{1 x} d_{1}+k_{2 x} d_{2}=0$, which is possible because $k_{x}$ is positive in conventional dielectrics and it is negative in left-handed materials. However, we find that this requirement is neither sufficient nor necessary to obtain complete band gaps.
In order to emphasize the importance of our findings presented below, first we recall the basic physics which explains why one-dimensional periodic structures containing materials of the same type (i.e., normal dielectrics) do not possess a complete three-dimensional band gap. Analyzing the effects associated with the wave scattering in Bragg gratings [13], we come to the conclusion that the only phenomenon which always allows for the wave propagation in the 1D dielectric periodic structures, and which cannot be suppressed by a choice of the structural parameters, is the waveguiding by optically dense layers. Indeed, it is well known that a dielectric waveguide with the core made of an optically dense medium always supports a fundamental mode. However, as was shown recently [14], the fundamental mode can be absent if the core is made of LH metamaterial. And it is this property of a LH waveguide that allows us to introduce a novel type of onedimensional periodic structures with a complete band gap.

Why does the usual dielectric waveguide always support the fundamental mode, and why a metamaterial waveguide does not? The condition for the guided waves to exist, defined by the dispersion relation for the modes in a slab waveguide, has a simple physical meaning: the round-trip accumulation of phase due to wave propagation across the layer, $2 \phi_{\text {prop }}$, including the phase retardation upon the total internal reflection, $2 \phi_{\text {refl }}$, should be equal to a multiple of $2 \pi$. The phase change due to the total internal reflection is negative for both types of waveguides, and depending on the angle of incidence it varies from 0 to $-\pi$. A difference between the conventional and LHM waveguides appears due to the phase accumulated by the wave propagating across the layer. In usual dielectrics, the wave is forward; i.e., the phase accumulated along the direction of energy flow is positive. As a result, there always exists an angle of wave propagation, such that the total phase change vanishes, and at least one mode always exists in a dielectric waveguide. In a LHM waveguide, the wave is backward, and the phase change $\phi_{\text {prop }}$ is negative. Then, one can choose the parameters in such a way that for all angles of wave propagation (greater than the angle of the total internal reflection), the total phase change is $-4 \pi<\phi=$ $2\left(\phi_{\text {refl }}+\phi_{\text {prop }}\right)<-2 \pi$, and no guided modes exist.

Based on the results presented above we can construct the one-dimensional periodic structure which possesses a complete band gap for one polarization. Indeed, we need to choose the layer thickness such that the guided modes are absent in the waveguides formed by the layers in the structure, and by varying the material parameters we should avoid the transmission resonances [13]. Our analysis shows that it is indeed possible to find such structures and, for example, the complete gap appears for the following set of parameters: $\epsilon_{1}=\mu_{1}=1, \epsilon_{2}=-6, \mu_{2}=$ $-1.38, \quad d_{1}^{(0)}=1.5 \lambda / 2 \pi, \quad d_{2}^{(0)}=1.4 \lambda / 2 \pi$, where $\lambda=$ $2 \pi c / \omega$ is the vacuum wavelength. Then we study the dependence of the band-gap spectrum on the structure period for a fixed ratio $d_{1} / d_{2}=d_{1}^{(0)} / d_{2}^{(0)}$, and Fig. 2(top) 

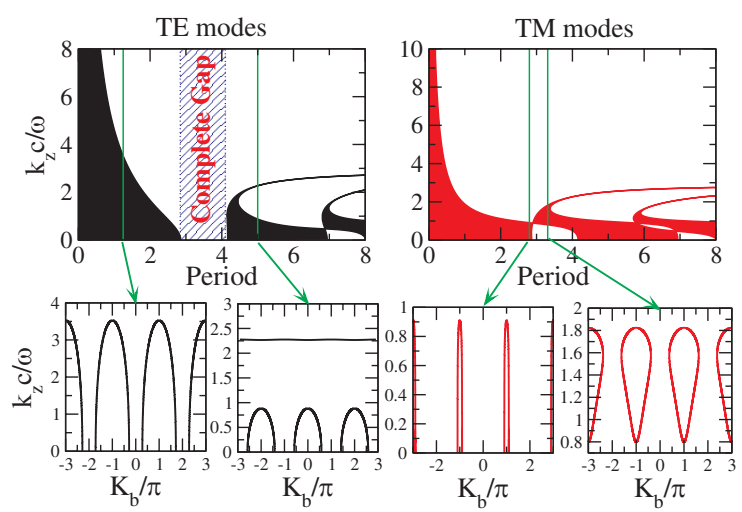

FIG. 2 (color online). (top) Shaded regions mark ranges of longitudinal wave numbers vs the structure period [normalized to $\lambda /(2 \pi)$ ] corresponding to TE (left) and TM (right) polarized electromagnetic waves that can propagate through the structure. (bottom) Dispersion diagrams of the Bloch waves for particular polarizations and periods, as indicated by arrows.

shows the bands (colored) where the wave propagation is possible. For the TE modes [see Fig. 2 (left, top)], there exists a range of periods for which the propagation is completely prohibited for all possible $k_{z}$, since the corresponding $K_{b}$ are complex. This means, in particular, that if we consider an end-fire generation problem and launch the waves along the layers towards the structure, they will be completely reflected, as schematically shown in Fig. 1(a). Such a regime is impossible for any type of the conventional dielectric gratings.

However, there is no complete band gap for the TMpolarized waves propagating in the same structure. In the optimal case with $d_{1,2} \approx d_{1,2}^{(0)}$, we have only one angle of propagation possible (i.e., the only value of $k_{z}$ with real $\left.K_{b}\right)$. This is the Brewster angle for which there is no reflection of TM waves at the interfaces. From the electromagnetic duality principle we find that taking the structure with LHM metamaterial characterized by $\epsilon_{2}^{\text {new }}=\mu_{2}$ and $\mu_{2}^{\text {new }}=\epsilon_{2}$, we can obtain a complete band gap for the TMpolarized waves. Most remarkably, the complete band gap for each polarization exists for rather broad ranges of the structure parameters; see Fig. 3. The regions for the complete TE and TM band gaps are symmetric with respect to the line $\epsilon_{2}=\mu_{2}$, and this is a consequence of the electromagnetic duality.

One-dimensional structures with a complete band gap for one of the polarizations can be used to form an electromagnetic cavity. To study the main features of the wave localization due to the presence of a complete band gap, we analyze the field of a line current $J$ running along the $y$ axis inside the structure at the position $\mathbf{r}_{0}=\left(x_{0}, z_{0}\right)$ in the $\mathbf{r}=(x, z)$ plane. It follows from the Maxwell's equations that the electric field can be expressed as $E(x, z)=$ $i \omega J \mu\left(x_{0}, z_{0}\right) G(x, z) / c^{2}$, where $G$ is the Green's function found as a solution of the following equation,

$$
\Delta G+\frac{\omega^{2}}{c^{2}} n^{2}(x) G-\frac{1}{\mu(x)} \frac{\partial \mu}{\partial x} \frac{\partial G}{\partial x}=4 \pi \delta\left(\mathbf{r}-\mathbf{r}_{0}\right) .
$$

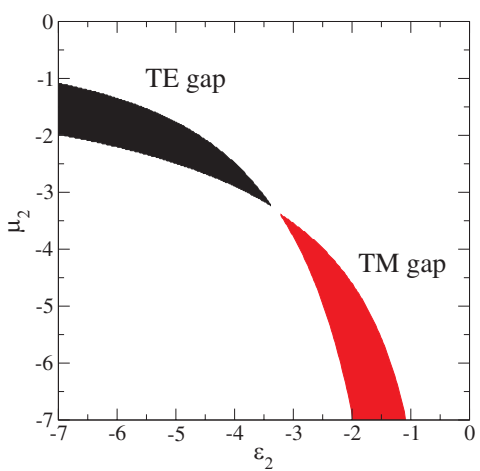

FIG. 3 (color online). Regions of parameters for which the complete TE (black) and complete TM (red) band gaps exist. The thickness $d_{1}^{(0)}$ and $d_{2}^{(0)}$ of the LH and RH layers are defined in the text.

The total emitted power per unit length of the line current $J$ is

$$
W=-\frac{\omega J^{2} \mu\left(x_{0}, z_{0}\right)}{2 c^{2}} \operatorname{Im}\left[G\left(x_{0}, z_{0}\right)\right],
$$

and this quantity is proportional to the local density of states (LDOS) [15]. The density of states (DOS) of the structure, which is an integral of LDOS over the Brillouin zone, characterizes the radiation efficiency of multiple sources located at different positions. The DOS becomes zero only if radiation in any direction in the plane is prohibited, indicated by the presence of a complete 2D band gap. We plot the dependence of the DOS on the magnetic permeability of negative-index material $\mu_{2}$ for fixed $\epsilon_{2}=-6$ and $d_{1,2}=d_{1,2}^{(0)}$ in Fig. 4 , which clearly demonstrates that a two-dimensional band gap exists for the TE-polarized waves within a certain range of media parameters. Within the band-gap region the radiation is

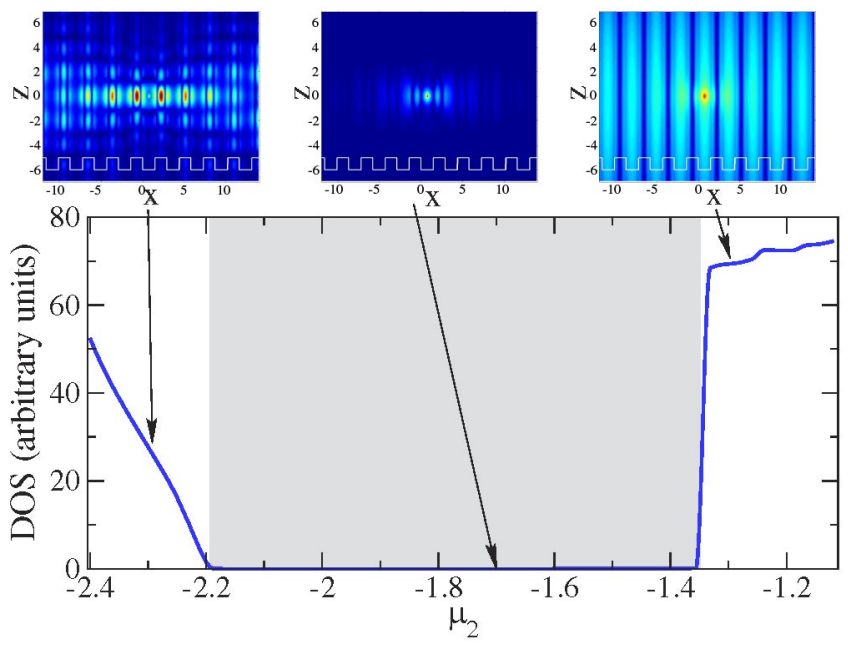

FIG. 4 (color online). Dependence of the two-dimensional density of states on the magnetic permeability of LHM. The top insets show the structure of the Green's function inside the band gap (middle), and outside the band gap (left and right), where the source position is $x_{0}=z_{0}=0$. 


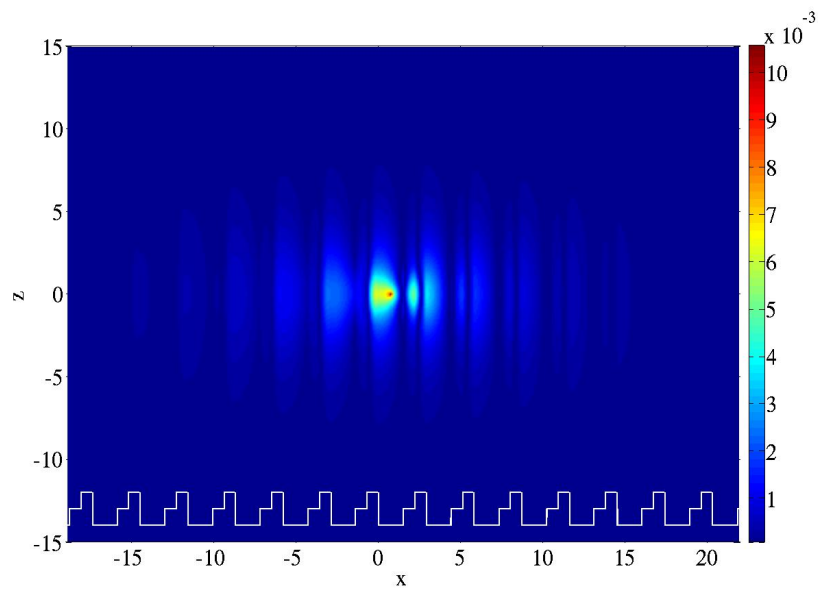

FIG. 5 (color online). The Green's function of a onedimensional three-layer periodic structure possessing an absolute band gap.

suppressed and the Green's function is exponentially localized for any source position [see Fig. 4 (top, middle)]. Outside the band gap, different propagating Bloch modes are excited, and the Green's function is not localized [see Fig. 4 (top, left and right)].

After the comprehensive study of the two-layer periodic systems and the properties of the complete band gaps supported by one-dimensional hybrid structures, we are able to suggest the case when the complete band gap may appear for both polarizations, thus allowing the existence of the absolute band gap. Indeed, to do this we should consider the more sophisticated case of a threelayer periodic structure in order to suppress the conditions for the existence of the Brewster angle which prevents us from creating a complete band gap in two-layer structures. The Brewster-angle transmission resonance can be easily eliminated by introducing a third layer in the structure, thus allowing the existence of a complete three-dimensional band gap for all waves propagating inside a specially designed one-dimensional structure. To demonstrate this quite unique property, we choose the structure with the parameters $\epsilon_{1}=\mu_{1}=1, \epsilon_{2}=\mu_{3}, \mu_{2}=\epsilon_{3}$, and $d_{2}=d_{3}$. The choice of this symmetry simplifies the analysis of the structure making it mathematically more elegant. Indeed, in this case the trace of the transfer matrix is the same for both polarizations, and it can be represented in the form

$$
\begin{aligned}
\operatorname{Tr}(M)= & 2 \cos \left(k_{1 x} d_{1}\right) \cos ^{2}\left(k_{2 x} d_{2}\right)-\left(\frac{\epsilon_{2}}{\mu_{2}}+\frac{\mu_{2}}{\epsilon_{2}}\right) \cos \left(k_{1 x} d_{1}\right) \\
& \times \sin ^{2}\left(k_{2 x} d_{2}\right)-\frac{1}{2}\left(\epsilon_{2}+\mu_{2}\right)\left(\frac{k_{1 x}}{k_{2 x}}+\frac{1}{\epsilon_{2} \mu_{2}} \frac{k_{2 x}}{k_{1 x}}\right) \\
& \times \sin \left(k_{1 x} d_{1}\right) \sin \left(2 k_{2 x} d_{2}\right) .
\end{aligned}
$$

Since the traces of the transfer matrices coincide for both TE and TM polarizations, the band gaps will appear in the structure spectrum for both the polarizations simultaneously. Existence of the transmission band gaps for both $\mathrm{TE}$ and TM-polarized waves in the same periodic structure indicate the existence of an absolute band gap. As an example, we show that the structure with the parameters $\epsilon_{2}=\mu_{3}=-6, \quad \epsilon_{3}=\mu_{2}=-1.38, \quad d_{1}=1.5 \lambda /(2 \pi)$, $d_{2}=d_{3}=0.7 \lambda /(2 \pi)$ possesses an absolute threedimensional band gap, and the Green's function corresponding to this three-layer structure is presented in Fig. 5.

In conclusion, we have revealed a novel and highly nontrivial property of left-handed metamaterials with negative refraction: a one-dimensional periodic structure containing layers made of a left-handed metamaterial can trap light in three dimensions due to the existence of a complete photonic band gap. This finding is in a sharp contrast with the fundamental concepts of the conventional physics of photonic crystals where complicated structures with two- and three-dimensional periodicity are required. We believe that our results suggest new directions for the future applications of metamaterials for microwaves, Terahertz frequencies, and visible light as fabrication technologies become available.

We thank C. Soukoulis, P. Belov, I. Gabitov, S. Mingaleev, V. Shalaev, and H. Winful for useful discussions.

[1] J. D. Joannopoulos, P. R. Villeneuve, and S. Fan, Nature (London) 386, 143 (1997).

[2] P. Lodahl, A. Floris van Driel, I. S. Nikolaev, A. Irman, K. Overgaag, D. Vanmaekelbergh, and W. L. Vos, Nature (London) 430, 654 (2004).

[3] P. Vukusic and J. R. Sambles, Nature (London) 424, 852 (2003).

[4] P. Yeh, Optical Waves in Layered Media (Wiley, New York, 1988).

[5] D. R. Smith, J. B. Pendry, and M. C. K. Wiltshire, Science 305, 788 (2004).

[6] K. Busch and S. John, Phys. Rev. E 58, 3896 (1998).

[7] J. N. Winn, Y. Fink, S. H. Fan, and J.D. Joannopoulos, Opt. Lett. 23, 1573 (1998).

[8] D. N. Chigrin, A. V. Lavrinenko, D. A. Yarotsky, and S. V. Gaponenko, Appl. Phys. A 68, 25 (1999).

[9] S. D. Hart, G. R. Maskaly, B. Temelkuran, P. H. Prideaux, J. D. Joannopoulos, and Y. Fink, Science 296, 510 (2002).

[10] I. Alvarado-Rodriguez, P. Halevi, and A. S. Sánchez, Phys. Rev. E 63, 056613 (2001); 65, 039901(E) (2002); J. R. Zurita-Sánchez and A.S. Sánchez, and P. Halevi, Phys. Rev. E 66, 046613 (2002); M. Wubs, L. G. Suttorp, and A. Lagendijk, Phys. Rev. E 69, 016616 (2004).

[11] J. Li, L. Zhou, C. T. Chan, and P. Sheng, Phys. Rev. Lett. 90, 083901 (2003).

[12] I. S. Nefedov and S. A. Tretyakov, Phys. Rev. E 66, 036611 (2002).

[13] P. St. J. Russel, T. A. Birks, and F. D. Lloyd Lucas, in Confined Electrons and Photons, edited by E. Burstein and C. Weisbuch (Plenum, New York, 1995), p. 585.

[14] I. V. Shadrivov, A. A. Sukhorukov, and Yu. S. Kivshar, Phys. Rev. E 67, 057602 (2003).

[15] P. Sheng, Introduction to Wave Scattering, Localization, and Mesoscopic Phenomena (Academic, Academic, 1995). 